\title{
La Cantantessa è una (e gli Studentessi sono solo canzonette) Paolo D'Achille
}

PUBBLICATO: 29 GENNAIO 2019

\section{Quesito:}

C.A., da Cagliari, ci chiede "se è lecito utilizzare la parola cantantessa, [...] ritenendo che la parolacantante sia sostantivo maschile e femminile".

\section{La Cantantessa è una (e gli Studentessi sono solo canzonette)}

Il quesito ripropone il problema della mozione (cioè del cambio di genere di un sostantivo in rapporto al sesso del referente), già affrontato varie volte dalla nostra consulenza (per esempio gli interventi di Cecilia Robustelli e di Paolo D'Achille su questo sito e quello di Anna M. Thornton sulla Crusca per voi n. 49) e più in generale la tematica del sessismo linguistico, che ha ormai una lunga tradizione di studi e su cui l'Accademia della Crusca è intervenuta più volte, anche di recente (si vedano l'intervento di Cecilia Robustelli nella sezioni "il tema" e i volumi di Claudio Marazzini e Giuseppe Zarra, "Quasi una rivoluzione». I femminili di professioni e cariche in Italia e allestero e di Cecilia Robustelli, con postfazione di Claudio Marazzini, Sindaco e sindaca. Il linguaggio di genere).

La nostra lettrice ha sicuramente ragione: i nomina agentis in -ante e-ente (molti dei quali si possono anche considerare conversioni da participi presenti) sono normalmente ambigeneri e quindi vengono usati tanto come maschili quanto come femminili. In passato, è vero, c'è stata una tendenza a formare dei femminili in-essa (brigantessa, presidentessa), anche perché la norma tradizionale prescriveva l'uso del suffisso-essa per formare femminili da basi maschili in -e (principe/principessa, dottore/dottoressa), oltre che in -a (poeta/poetessa). Ma l'uso di questo suffisso, che è stato aggiunto, inutilmente sul piano morfologico, anche a nomi maschili in -o, spesso con una decisa connotazione spregiativa o ironica (medichessa, deputatessa, ecc.), è stato poi considerato discriminatorio nell'ottica del sessismo linguistico. Così, alle forme sopra citate si sono spesso affiancate quelle in cui la distinzione di genere è lasciata all'articolo e alle altre modalità di accordo grammaticale, come la presidente, che è da considerare ormai la forma standard. Mi sembra tuttavia di cogliere oggi una certa ripresa di presidentessa, che in passato indicava la moglie del presidente, che ho sentito più volte usare di recente (Paola Villani, Le donne al parlamento. Genere e linguaggio politico, in Per Tullio De Mauro. Studi offerti dalle allieve in occasione del suo $80^{\circ}$ compleanno, a cura di Anna M. Thornton, Miriam Voghera, Roma, Aracne, 2012, pp. 317-339, segnala però che presidentessa ha ambiti di riferimento meno "alti" e si usa "per designare o donne che presiedono associazioni, enti, club sportivi o capi di Stato e di Governo straniere" oppure "ha connotazioni ironiche o spregiative", p. 330).

Con cantantessa ci troviamo di fronte a un caso particolare. La parola è registrata tra i Neologismi quotidiani. Un dizionario a cavallo del millennio 1998-2003 di Giovanni Adamo e Valeria Della Valle (Firenze, Olschki, 2003), ma si riferisce in realtà esclusivamente a una ben precisa artista, la cantautrice siciliana Carmen Consoli, nel cui sito ufficiale si leggeva fino a qualche tempo fa la seguente spiegazione del termine (regolarizzo l'uso di apostrofi e accenti):

Tutto successe nel periodo d'incisione, nello studio con lingegnere del suono sud africano Allan Goldberg. Carmen con un suo amico cercava di far capire l'uso del suffisso 'essa' per il femminile. Cosi fecero un esempio per farglielo capire meglio, prendendo come cavia un cane di nome Mela e gli dissero che in italiano il Cane femmina si chiamava Canessa. Così Allan Goldberg sentendo che Cantante per Carmen suonava male, utilizzò l'appellativo Cantantessa.

Anche su Wikiquote si riporta il passo di un'intervista in cui la cantautrice riferisce la notizia:

Fu un errore di un ingegnere del suono sudafricano che voleva dire di stare zitti perché la cantante doveva cantare, ma sembrandogli di rivolgersi a un uomo disse "la cantantessa". Mi piace perché non è un termine serio e non vale come dire "la cantante", quella che sa cantare. Io invece voglio passare come una che canta, una cantantessa appunto, che sta un gradino più sotto. 
Dunque cantantessa è da considerare quasi un nome proprio, un soprannome antonomastico, se pure particolare (non tratto direttamente da un nome comune), e non a caso nel primo passo sopra riportato figura con l'iniziale maiuscola. Ipotizzerei che Carmen Consoli abbia accettato di essere definita "cantantessa" non solo per i motivi, apparentemente "autodenigratori", esposti nell'intervista, ma anche perché il nome si presta a essere letto pure come parola macedonia, formata da cantant(e) + (po)etessa, considerando l'importanza che nelle sue canzoni assume la componente verbale, il testo.

Vero è che, di recente, la parola è stata talvolta usata anche al plurale, come dimostrano questi due esempi, nel primo dei quali cantantesse sembra significare 'cantatrici' (e, data l'ambientazione settecentesca del testo, potrebbe arieggiare formazioni in -essa ormai desuete, come la mercantessa di manzoniana memoria), nel secondo 'cantautrici' (con implicito riferimento a Carmen Consoli):

Nobili e cardinali, fraticelli mendicanti, gesuiti; artisti e mercanti d'arte, pastori arcadici; pie matrone e famose cantantesse, ugole doro; ambasciatori d'ogni corte d'Europa, dogni paese (Vittorio Giacopini, Nello specchio di Cagliostro. Un sogno a Roma. Romanzo, Milano, il Saggiatore, 2013).

[...] brave artiste, dai timbri unici e dotate di grandi e diversificate capacità interpretative, dubitavo fortemente che ci fosse bisogno di cantantesse straniere... (Giacomo Lucchesi, La musa salvifica (1976-1981), 2017, e-book).

Posso aggiungere un'attestazione metalinguistica, tratta dalla conclusione della prima circolare firmata da Anna M. Thornton nella sua veste di Presidente della Società di Linguistica Italiana, pubblicata sul "Bollettino SLI" 2/2015:

Infine, una piccola nota linguistica. Emanuele Banfi, nel rivolgermi per primo le congratulazioni per la mia nomina, mi ha chiesto se avrei voluto essere chiamata "il presidente" o "la presidentessa": ebbene, né l'uno né l'altro, vorrei essere chiamata "la presidente", come da anni suggeriscono le Raccomandazioni per un uso non sessista della lingua italiana. Sono ben consapevole che gli usi linguistici privati e informali non si impongono per legge, ma mi fa piacere rispondere a chi mi chiede la mia preferenza. Chi trova strana la formula "la presidente" si chieda: Mina è "un cantante" o "una cantantessa"?

Per restare nell'ambito della musica leggera, vorrei ricordare che Elio e le Storie Tese, un gruppo che ama spesso ricorrere all'ironia e al nonsense, hanno intitolato un loro album del 2008 Studentessi, volgendo al maschile il femminile plurale di studente. In rete si legge (cfr. la voce di Wikipedia) che il titolo dell'album sarebbe una "citazione" tratta da un'intervista rilasciata da Ilona Staller, pornoattrice ungherese molto popolare in Italia negli anni Settanta come Cicciolina, che fu anche eletta al Parlamento come deputata (qualche maligno direbbe deputatessa). In ogni caso, la forma è notevole, anche perché, data la tendenza, rilevata all'inizio, al rifiuto del suffisso-essa, accanto a studentessa si è diffusa la forma la studente (che tuttavia mi risulta di uso circoscritto, in ambienti apertamente femministi e/o nei corsi di italiano all'estero).

Se sono veri entrambi gli aneddoti sopra riportati, la coniazione sia di cantantessa sia di studentessi sarebbe da attribuire a stranieri e a loro "errori" di italiano. Ma cantantessa, come si è detto, ha dei precedenti nella storia dell'italiano e retroformazioni come studentessi, in ambienti di lavoro in cui la componente femminile è prevalente, sono ben possibili, e non solo come occasionalismi scherzosi: più di un insegnante delle secondarie inferiori mi ha detto di essere stato interpellato da propri alunni (siamo dunque ancora in una fase di apprendimento della lingua) come professoresso!

In definitiva, i nostri cantantessa estudentessi vanno considerati nomi propri (un soprannome, il titolo di un album) o tutt'al più occasionalismi. La forma femminile di cantante è soltanto (la) cantante, mentre la coppia studente/studentessa resta ancora maggioritaria nell'italiano di oggi.

\section{Cita come:}

Paolo D'Achille, La Cantantessa è una (e gliStudentessi sono solo canzonette), "Italiano digitale", 2019, VIII, 2019/1 (gennaio-marzo) , pp. 13-15.

DOI: $10.35948 / 2532-9006 / 2019.3063$ 
Copyright 2019 Accademia della Crusca

Pubblicato con licenza creative commons CC BY-NC-ND 